\title{
Modeling the cognitive trajectory in CADASIL
}

Sandrine Brice, Aude Jabouley, Sonia Reyes, Carla Machado, Christina Rogan, Nathalie Dias-Gastellier, Hugues Chabriat, Sophie Tezenas du Montcel

Author affiliations

Sorbonne Université, INSERM, Institut Pierre Louis d'Épidémiologie et de Santé Publique, F75013 Paris, France (Sandrine Brice)

Département de Neurologie, Centre de référence CERVCO, Hôpital Lariboisière, AP-HP, Université Paris 7 Denis Diderot, 75010 Paris, France (Aude Jabouley, Sonia Reyes, Carla Machado, Christina Rogan, Nathalie Gastellier, Hugues Chabriat)

INSERM, Unité Mixte de Recherche 1161, 75010 Paris, France (Hugues Chabriat)

Sorbonne Université, INSERM, Institut Pierre Louis d'Épidémiologie et de Santé Publique, AP-HP, Hôpitaux Universitaires Pitié Salpêtrière - Charles Foix, F75013 Paris, France (Sophie Tezenas du Montcel)

Present address of Christina Rogan: GHU Paris psychiatrie \& neurosciences, 1 rue Cabanis, 75014 Paris

Corresponding author: Sophie Tezenas du Montcel

Postal address: Département d'information médicale (DIM) - GHU APHP Sorbonne Université site Pitié Salpêtrière, 47-83 boulevard de l'Hôpital, 75651 Paris cedex 13, France Phone number: $+33(0) 142160582$

E-mail address: sophie.tezenas@aphp.fr 


\section{ABSTRACT AND KEYWORDS}

\section{Abstract}

\section{Background}

For developing future clinical trials in Cerebral autosomal dominant arteriopathy with subcortical infarcts and leukoencephalopathy (CADASIL), it seems crucial to study the long term changes of cognition.

\section{$\underline{\text { Objective }}$}

We aimed to study the global trajectory of cognition, measured by the Mini-Mental State Examination (MMSE) and the Mattis Dementia Rating Scale (MDRS), along the course of CADASIL.

\section{$\underline{\text { Methods }}$}

Follow-up data of 185 CADASIL patients, investigated at the French National Referral center CERVCO as from 2003, were considered for analysis based on strict inclusion criteria. Assuming that the MMSE and the MDRS provide imprecise measures of cognition, the trajectory of a common cognitive latent process during follow-up was delineated using a multivariate latent process mixed model. After adjustment of this model for sex and education, the sensitivities of the two scales to cognitive change were compared.

\section{$\underline{\text { Results }}$}

Analysis of the cognitive trajectory over a time frame of 60 years of age showed a decrease of performances with aging, especially after age of 50 years. This decline was not altered by sex or education but patients who graduated from high school had a higher mean cognitive level at baseline. The sensitivities of MMSE and MDRS scales were similar and the two scales suffered from a ceiling effect and curvilinearity. 


\section{Conclusion}

These data support that cognitive decline is not linear and mainly occurs after the age of 50 years during the course of CADASIL. They also showed that MMSE and MDRS scales are hampered by major limitations for longitudinal studies.

\section{Key words}

CADASIL; Cognitive Decline; Longitudinal Studies; Neuropsychological Tests; Latent Variable Modeling; Patient Outcome Assessment; Aging; Behavior; Alzheimer's disease 


\section{INTRODUCTION}

Cerebral autosomal dominant arteriopathy with subcortical infarcts and leukoencephalopathy (CADASIL), caused by mutations of the NOTCH3 gene on chromosome 19, is a unique model of pure vascular dementia to investigate the natural history of severe ischemic small vessel diseases [1]. The main symptoms of CADASIL include attacks of migraine with aura, stroke events, mood and behavior troubles, gait disturbances, and cognitive decline from executive dysfunction up to severe dementia [2]. Accumulating data showed that disability, particularly cognitive decline from the early stage, can progress in CADASIL in the absence of ischemic or hemorrhagic stroke events. Therefore, cognitive decline should be obviously considered as a key outcome in future clinical trials in CADASIL in addition to the occurrence of stroke events.

Yet, the selection of cognitive scales for evaluating the actual longitudinal changes of cognitive performances in CADASIL is not trivial. Many aspects should be considered for selecting the most relevant tools. First, disease progression is observed over several decades and may not be linear. Recent data support that progression of disability along aging can differ at individual level according to the stage of the disease. Second, factors such as sex or mostly education may largely impact cognitive performances. Previous data support that females may have better performances than males, particularly at early stage, in CADASIL [3-6]. Also, more generally, highly educated subjects have better performances on a wide range of cognitive tasks included in different global scales [7-9]. Finally, measures of cognitive changes in longitudinal studies also depend largely on the neuropsychological test itself. In order to capture cognitive changes with enough accuracy, a neuropsychological test should be able not only to explore the different cognitive domains specifically affected during the progression of the disease but also to measure these changes in the range of cognitive levels observed in the targeted population. This capacity might be strongly limited by several 
metrological properties of tests such as the presence of a ceiling effect as observed using the Mini-Mental State Examination (MMSE) [10] in Alzheimer's and Parkinson's diseases [1113]. Potential curvilinearity may also represent another major limitation when a fixed loss of points does not correspond to the same intensity of cognitive decline over the whole range of the test values. In CADASIL, global cognitive performances were previously assessed longitudinally using various global cognitive scales such as the MMSE or the Mattis Dementia Rating Scale (MDRS) [14]. The MMSE is widely used to screen cognitive impairment in daily clinical practice. The MDRS is an alternative test for evaluating global cognitive impairment in clinical settings including disorders affecting primarily executive functions. In CADASIL, significant variations of the corresponding scores were previously detected over 3 years [15]. However, the overall trajectory of cognitive decline during the whole disease progression and the actual factors that may influence this evolution remain poorly understood, even though this information is crucial for the timely use of cognitive testing in longitudinal studies. Indeed, the profile of cognitive deficits in CADASIL has been previously investigated in four major studies [16-19] mainly focused on variations of performances in different specific cognitive domains. However, the global cognitive level during the course of the disease was not investigated yet.

In the present study, we aimed to investigate the global trajectory of cognitive decline during the course of CADASIL, a model of vascular dementia related to cerebral small vessel disease, over several decades and compare the metrological properties of MMSE and MDRS scales for longitudinal studies. For this purpose, an original analytical approach was used to take into account curvilinearity and ceiling effect of the two scales. Curvilinear mixed models, which can be seen as extended linear mixed models [20], were used to investigate both the change over time of the latent cognitive process in CADASIL and the sensitivity to cognitive change of the two scales. 


\section{MATERIALS AND METHODS}

\section{Study population}

Between 2003 and 2017, a total of 366 patients were recruited at the French National Referral center CERVCO (https://www.cervco.fr/) in a large prospective cohort of CADASIL patients. Inclusion criteria in the cohort were being at least 18 years of age, with a documented mutation in the NOTCH3 gene, and willing to be regularly evaluated. Neuropsychological assessment was obtained at inclusion and every 20 months of follow-up in median. Reasons for dropout of patients during follow-up were not recorded. Dedicated methods were used for database verification, cleaning, and validation.

Informed consent was obtained from each subject or from a close relative when the patient was too severely disabled to give written consent. The study was approved by an independent ethics committee.

\section{Assessment of cognition}

Cognitive performances were assessed at each visit using both the MMSE and the MDRS. The MMSE is a global neuropsychological test evaluating the following dimensions of cognition: orientation, memory, attention, language, and constructional praxis. The sum score on the MMSE ranges from 0 (worse) to 30 (best). The MDRS consists of five subscale scores related to attention, initiation-perseveration, construction, conceptualization, and memory. The MDRS total score ranges from 0 (worse) to 144 (best). When a subtest could not be applied because of too severe cognitive alterations, the patient was considered to have altered performance for this subtest. The lowest score for this subtest was then used for this specific performance. Raw total scores were analyzed. 


\section{Explanatory variables}

The following parameters considered as potential predictors of clinical worsening in CADASIL were available at baseline: age, sex, education, smoking, alcohol consumption, hypertension, diabetes, systolic blood pressure, diastolic blood pressure, homocysteine, previous stroke events, gait disturbances, balance problems, disability, and dementia. Education was determined according to the French Barbizet scale [21] and summarized into a binary covariate as 1 when the subject passed at least the high school diploma (high level) and 0 otherwise (low level). Alcohol consumption was a qualitative variable with three categories: never, $<2$ glasses of wine for a man ( 1 glass or equivalent for a woman), and $>2$ glasses of wine for a man (1 glass or equivalent for a woman). Previous stoke events were considered to be transient ischemic attacks if they lasted less than 24 hours. Disability was assessed using the modified Rankin Scale (mRS) and was turned into a binary covariate corresponding to 1 when the subject had a score of 3 or more (moderate or severe disability) and to 0 otherwise (no disability). Dementia was assessed according to DSM-IV criteria. Age was available throughout the follow-up and considered as the time variable. For computation purposes, the time scale was the age minus 24 years per 10 years where 24 corresponds to the age of the youngest patient observed at baseline. In this way, the intercept represents the cognitive level at 24 years of age and the change in cognitive level is measured for a decade. Neuroimaging data were not available.

\section{Statistical analysis}

\section{Descriptive statistics}

Data were first summarized at baseline, with frequencies and percentages for qualitative data and means and standard deviations (SD) for quantitative variables. 


\section{Modeling of the cognitive trajectory}

We used mixed models for curvilinear outcomes [20]. They are divided in two parts: (i) a linear mixed model which describes the change over time of the latent cognitive process and evaluates the common effects of covariates on this latent cognitive trajectory; (ii) test-specific nonlinear measurement models which relate each neuropsychological assessment with the latent cognitive process and allows determining metrological properties of the different neuropsychological tests and evaluating test-specific associations with covariates after adjustment for their effect on the latent cognitive process (contrasts). In the model involving both the MMSE and the MDRS, the latent cognitive process captures the common cognitive part measured by the neuropsychological tests. The latent process constitutes the actual unobserved cognitive level that generated the scores on the MMSE and the MDRS and does not have a particular clinical meaning.

First, we analyzed the MMSE and the MDRS separately. The change over time of the latent process underlying each score was described using a cubic function of time with correlated random effects on the intercept, slope, quadratic slope, and cubic slope. The objective was to select the best parameterized function linking each sum score to its underlying latent process. For this purpose, we estimated latent process mixed models for univariate longitudinal data assuming a similar trajectory of the underlying latent process with a different link function for each model. We compared eight link functions according to the Akaike information criterion (AIC): linear transformation, beta $\mathrm{CDF}$, and quadratic Isplines with three, five or seven nodes located at the quantiles of the neuropsychological test distribution or equidistant.

Second, we used a latent process mixed model for multivariate longitudinal data to analyze the cognitive trajectory. We assumed that MMSE and MDRS tests are measures with error of the same underlying latent process corresponding to global cognitive functioning. In 
order to link the common latent process with each neuropsychological test, we used the transformations previously selected to link each test with its underlying latent process. We then ascertained the shape of the cognitive trajectory. To test whether the addition of a random effect improves the model fit, we compared nested models using an approximation of the distribution of the likelihood ratio statistic as proposed by Stram and Lee [22]. We used the AIC to select the best variance-covariance structure of the random effects between unstructured and diagonal and to ascertain the need for an autocorrelated Gaussian process. To test whether an additional fixed effect was different from 0 , nested models were compared using the likelihood ratio test (LRT). The AIC was used to determine whether test-specific random effects should be added.

The model was adjusted for sex and education since cognitive performances have been previously related to these factors [3-9]. Interactions between sex and time and between education and time were tested using the LRT. Adequacy of the model to data was checked with plots, namely marginal residuals versus marginal predictions and subject-specific residuals versus subject-specific predictions. The assumption of normality of marginal and subject-specific residuals was evaluated with QQ plots (data not shown). All other potential predictors of clinical worsening in CADASIL were then considered one by one using the LRT.

Statistical tests were performed at the conventional two-tailed type I error of 0.05 . Data were analyzed using R version 3.4.0 [23]. Nonlinear mixed models for longitudinal data were estimated using the package 'lcmm' [24]. 


\section{RESULTS}

\section{Sample selection}

Only individuals having at least three visits with complete results for both MMSE and MDRS tests were selected. A total of 841 observations were collected from 185 patients with a median number of four observations available per individual (interval quartile range (IQR): 3-6). The numbers of observations and patients per age range are given in Supplementary Table 1 in the Supplementary Tables. The follow-up duration varied from 3 to 14 years with a median follow-up interval of 20 months (IQR: 18-25). These patients were younger and less severe than those who were not included in the analysis as they significantly suffered less from hypertension, gait disturbance, balance problems, disability, and dementia at inclusion and had a significantly lower homocysteine level and significantly higher scores on the MMSE and the MDRS at inclusion (Table 1). They also tended to suffer less from diabetes than the patients who were not included in the analysis.

\section{Descriptive statistics}

Among the 185 patients, $43.2 \%$ were men and $41.6 \%$ had passed their high school diploma (Table 1). At baseline, the mean scores of MMSE and MDRS tests were 27.8 and 137.8 respectively. The patients were aged 50.3 years on average (SD: 11.5; IQR: 42.3-59.3). The distributions of the two neuropsychological tests (total scores) were strongly skewed to the left (Supplementary Figure 1 in Supplementary Figures). The maximum values of MMSE and MDRS tests were reached respectively in about $34 \%$ and $21 \%$ of the data, indicating a strong ceiling effect for these two neuropsychological tests. The observed mean evolutions are given in the additional material (Supplementary Figure 2 in Supplementary Figures). 


\section{Choice of the link functions}

For each total score, eight univariate latent process mixed models including each a different link function were tested (Supplementary Table 2 in the Supplementary Tables), without adjustment for covariates since we aimed to analyze the relationship between each score and its underlying latent process. According to the AIC, the quadratic I-splines with three nodes located at the quantiles of the MMSE distribution and the quadratic I-splines with five nodes located at the quantiles of the MDRS distribution provided the best fits.

\section{Cognitive evolution with age}

We modeled the trajectory of the latent process underlying the MMSE and the MDRS using the two best link functions to link each score with the common latent process. The shape of the cognitive trajectory resulted in a quadratic function of time with correlated random effects on the intercept, slope, and quadratic slope to account for inter-individual variability (Supplementary Table 3 in the Supplementary Tables). The quadratic shape suggests an acceleration of cognitive decline at older ages (Figure 1). The intercept represents the cognitive level at 24 years of age in the reference category, corresponding to women who did not obtain the high school diploma, and the slope and the quadratic slope measure the change in cognitive level for a decade. The model was improved by a Brownian motion accounting for the within-subject variability ( $\Delta$ AIC $=-5.91$ points) and the addition of test-specific random effects $(\Delta \mathrm{AIC}=-41.74$ points), which implies that for a same value of latent cognition, subjects score differently on the neuropsychological tests. The model was then adjusted for sex and education.

The estimates and standard errors (SE) related to the fixed effects parameters are shown in Table 2. Sex was not significantly associated with the mean cognitive level at baseline $(p=0.136)$. In contrast, subjects who graduated from high school had a significantly 
better mean cognitive level at baseline $(\hat{\beta}=1.01 \pm 0.43$ (SE) unit of the latent cognitive process at baseline per decade of age, $p=0.019$ ) than subjects who did not. The effects of sex and education did not differ from test to test (contrasts: $p=0.085 ; p=0.283$ ). The percentage of variance explained by the common latent process at a given time for each neuropsychological test was age-dependent and increased with age (Supplementary Figure 3 in the Supplementary Figures). The latent process explained 37\%, 44\%, and $51 \%$ of the variance of the MMSE at age 46 years, 54 years, and 63 years respectively. For the MDRS, the percentage of variance explained by the latent process was $65 \%$ at age 46 years, $71 \%$ at age 54 years, and $77 \%$ at age 63 years.

\section{Influence of baseline characteristics on cognitive evolution}

The estimated interactions between age at inclusion (per 10 years increase) and the slope and between age at inclusion and the quadratic slope were respectively $0.60 \pm 0.23(\mathrm{SE})$ and $0.02 \pm 0.03(\mathrm{SE})(p<0.001)$ (Table 3), which suggests that age at inclusion impacts the change in cognitive functioning during follow-up. No other significant interaction was detected between the different covariates and time. In particular, the effects of sex and education on the change of cognitive level related to age were not significant (interactions: $p$ $=0.104 ; p=0.344)$.

\section{Sensitivity to cognitive change of the MMSE and the MDRS}

The estimated link functions between each neuropsychological test and the common underlying latent process are displayed in Figure 2. The two neuropsychological tests showed a nonlinear transformation: a one-point change in the natural scale of the MMSE and the MDRS at high levels of cognition corresponds to a greater decrease in cognitive level than a one-point change in the natural scale of the MMSE and the MDRS at low levels of cognition, 
which highlights the curvilinearity of these two tests. The shape of the tests at high cognitive levels was horizontal: the neuropsychological tests do not allow identifying small cognitive changes at high levels of cognition, which attests to their ceiling effect. The confidence intervals of both link functions largely overlapped (Figure 2), suggesting that the two transformations are very similar. However, the values in the latent process scale vary between -5.46 and 2.34 for the MMSE and from -5.07 to 2.00 for the MDRS. In the latent process scale, the range of values of the MDRS is included in that of the MMSE and represents $91 \%$ of the range of values of the MMSE.

\section{DISCUSSION}

The results of this study allowed for the first time to delineate a global trajectory of cognitive decline during the course of CADASIL. This was obtained with a dedicated analytical model and with pooled individual evolutions from a large amount of cognitive data obtained from 185 patients of different age who were followed-up regularly for 3 to 14 years. Globally, a decrease in MMSE and MDRS total scores was observed in relation to cognitive decline developing with age, especially after 50 years. An increase of scores related to the latent cognitive process was also detected on the initial trajectory until approximately the age of 40 years. Such an increase may result from a first re-test effect as already described in studies of normal aging [25]. Indeed, better scores can be obtained at the second visit due to stressinduced effects on cognitive performances at the initial visit and to possible learning effects between the first two visits. In our results, we did not find such an effect and hypothesized that this initial increase of the latent cognitive level was rather related to the quadratic shape of the modeled trajectory itself. The model was adjusted for sex and education although no significant effect of sex or education was detected on the cognitive decline. Only a difference of cognitive level was observed at baseline for individuals with the highest level of education. 
These results are concordant with previous data obtained in healthy elderly subjects or in patients with Alzheimer's disease showing that a higher educational level is associated with a higher latent cognitive level at baseline [20,26]. Contrasts for these two covariates on the latent cognitive level derived from MMSE or MDRS tests were also not found significant.

Our analysis also allowed investigating the potential impact of different covariates obtained at baseline on the global cognitive trajectory of the disease. Neither sex nor education level was found to influence the global course of cognition, which suggests that these variables should not be necessarily considered for future analysis of longitudinal data in CADASIL. Only age at baseline had a significant effect on the course of cognitive decline. These results are in line with those obtained on the latent cognitive decline along aging in the PAQUID study or in Alzheimer's disease patients [20,26]. In contrast with previous results obtained in a 3-year follow-up study, we did not find either any effect of gait disturbances, balance problems or dementia on the global cognitive decline trajectory. This discrepancy might be related to our inclusion criteria in the present study which may have caused a survival bias. Herein, patients included in the analysis had to complete both MMSE and MDRS tests at three visits at least and were therefore less likely to have motor difficulties occurring at the latest stage of the disease and severe dementia. Furthermore, all patients were selected from a cohort of individuals originating from multiple regions in France and who had to travel to the referral center for their clinical evaluation, which may have prevented the inclusion of patients presenting with the most severe motor difficulties. Interestingly, no significant effect of the traditional vascular risk factors was found on the global cognitive trajectory. However, we cannot exclude some age-dependent effects of cardiovascular risk factors on cognitive performances as already reported in large epidemiological studies with aging [27] since our models were adjusted for baseline covariates instead of time-varying covariates. Unfortunately, the results lack of neuroimaging data which would complete the 
study. Furthermore, there was presumably limited statistical power to evaluate some of the hypotheses tested with no more than 185 patients included in the analysis and a model that estimates many parameters. In addition, the results may be influenced by the type of neuropsychological tests used in the analysis.

The selection of a limited number of measures for longitudinal studies of cognition is crucial as previously investigated in healthy elderly subjects [28] or in Alzheimer's disease patients [29]. Major limitations were observed using the MMSE and the MDRS for evaluating cognitive decline in CADASIL. Both showed an obvious problem of curvilinearity which could have major consequences on the results of clinical trials. Indeed, due to the varying sensitivity to cognitive change of the MMSE and the MDRS, it will be complicated to assess the benefits of a treatment for cognitive impairment in CADASIL patients with no or mild cognitive impairment using these tests because an identical decrease in scores on the MMSE and the MDRS results in a more or less important decrease in cognitive level depending on the initial scores of the patients on these tests. The two neuropsychological tests also have a problem of ceiling effect for longitudinal studies as already shown for the MMSE when measuring the rate of cognitive changes in dementia with Lewy bodies [13]. The two estimated transformations were finally very close although MDRS score changes were explained by a larger percentage of the latent cognitive variation.

The strength of this study relates to the methodology adopted for longitudinal analysis of MMSE and MDRS scores. Our analysis considered the characteristic properties of these tests which are often ignored such as their ceiling effect and curvilinearity. We initially verified that non-linear transformations provided much better fitting for both MMSE and MDRS scores than the use of a standard linear transformation (data not shown). The amount of data collected in CADASIL patients aged from 24 to 82 years is also an exceptional source for conducting such an analytical approach. There are also limitations for interpreting the 
present results because it is complicated to characterize the latent process which is defined according to the pool of neuropsychological tests included in the analysis. The link functions were selected from the univariate models (Supplementary Table 2 in the Supplementary Tables) and used in the multivariate model with a different latent process than those of the univariate models. However, since the MMSE and the MDRS are both a measure of global cognition, it is reasonable to interpret the modeled latent cognitive process as a global cognitive factor. Finally, our analysis was based on the sum scores using the MMSE and the MDRS as often used in clinical practice [30,31]. Different results cannot be excluded using subscores derived from these two global scales.

In conclusion, this study strongly supports that patients aged around 50 years of age should be selected preferentially for future therapeutic clinical trials that aim to reduce cognitive decline in CADASIL. It also indicates that both the MMSE and the MDRS have important limitations including curvilinearity and ceiling effect which limit their use in patients with a large range of cognitive deficits or in the healthier or highly educated subjects. The results obtained with such scales should be interpreted with caution. Additional studies are needed to improve the available armamentarium for evaluating cognitive decline in longitudinal studies of progressive ischemic small vessel disease as CADASIL.

\section{ACKNOWLEDGMENTS (INCLUDING SOURCES OF SUPPORT)}

The authors acknowledge all patients who participated in this research, their families and the association CADASIL France (http://www.cadasil.fr/) for their active collaboration. They also thank Pr. Sylvie Chevret, Claire Pacheco, and Abbas Taleb for their investment in the preparation of the database. This study was supported by grants from the RHU TRT_cSVD (https://treat-svd.fr/). 


\section{CONFLICT OF INTEREST/DISCLOSURE STATEMENT}

Hugues Chabriat received fees from Servier and Hovid Companies during the study period for participating in Steering Committees of clinical trials unrelated to this research. 


\section{REFERENCES}

[1] Tournier-Lasserve E, Joutel A, Melki J, Weissenbach J, Lathrop GM, Chabriat H, Mas JL, Cabanis EA, Baudrimont M, Maciazek J (1993) Cerebral autosomal dominant arteriopathy with subcortical infarcts and leukoencephalopathy maps to chromosome 19q12. Nat. Genet. 3, 256-259.

[2] Chabriat H, Joutel A, Dichgans M, Tournier-Lasserve E, Bousser M-G (2009) CADASIL. The Lancet Neurology 8, 643-653.

[3] Collaer ML, Hines M (1995) Human behavioral sex differences: a role for gonadal hormones during early development? Psychol Bull 118, 55-107.

[4] Kramer JH, Delis DC, Kaplan E, O’Donnell L, Prifitera A (1997) Developmental sex differences in verbal learning. Neuropsychology 11, 577-584.

[5] Reite M, Cullum CM, Stocker J, Teale P, Kozora E (1993) Neuropsychological test performance and MEG-based brain lateralization: sex differences. Brain Res. Bull. 32, $325-328$.

[6] Wiederholt WC, Cahn D, Butters NM, Salmon DP, Kritz-Silverstein D, BarrettConnor E (1993) Effects of age, gender and education on selected neuropsychological tests in an elderly community cohort. J Am Geriatr Soc 41, 639-647.

[7] Jorm AF, Scott R, Henderson AS, Kay DW (1988) Educational level differences on the Mini-Mental State: the role of test bias. Psychol Med 18, 727-731.

[8] Launer LJ, Dinkgreve MA, Jonker C, Hooijer C, Lindeboom J (1993) Are age and education independent correlates of the Mini-Mental State Exam performance of community-dwelling elderly? J Gerontol 48, P271-277.

[9] Tombaugh TN, McIntyre NJ (1992) The mini-mental state examination: a comprehensive review. J Am Geriatr Soc 40, 922-935. 
[10] Folstein MF, Folstein SE, McHugh PR (1975) "Mini-mental state". A practical method for grading the cognitive state of patients for the clinician. J Psychiatr Res 12, 189198.

[11] Hoops S, Nazem S, Siderowf AD, Duda JE, Xie SX, Stern MB, Weintraub D (2009) Validity of the MoCA and MMSE in the detection of MCI and dementia in Parkinson disease. Neurology 73, 1738-1745.

[12] Trzepacz PT, Hochstetler H, Wang S, Walker B, Saykin AJ, Alzheimer's Disease Neuroimaging Initiative (2015) Relationship between the Montreal Cognitive Assessment and Mini-mental State Examination for assessment of mild cognitive impairment in older adults. BMC Geriatr 15, 107.

[13] Biundo R, Weis L, Bostantjopoulou S, Stefanova E, Falup-Pecurariu C, Kramberger MG, Geurtsen GJ, Antonini A, Weintraub D, Aarsland D (2016) MMSE and MoCA in Parkinson's disease and dementia with Lewy bodies: a multicenter 1-year follow-up study. J Neural Transm (Vienna) 123, 431-438.

[14] Mattis S (1976) Mental Status examination for organic mental syndrome in elderly patients. In Geriatric Psychiatry: A Handbook for Psychiatrists and Primary care Physicians Grune \& Stratton, New-York.

[15] Jouvent E, Duchesnay E, Hadj-Selem F, De Guio F, Mangin J-F, Hervé D, Duering M, Ropele S, Schmidt R, Dichgans M, Chabriat H (2016) Prediction of 3-year clinical course in CADASIL. Neurology 87, 1787-1795.

[16] Amberla K, Wäljas M, Tuominen S, Almkvist O, Pöyhönen M, Tuisku S, Kalimo H, Viitanen M (2004) Insidious cognitive decline in CADASIL. Stroke 35, 1598-1602.

[17] Peters N, Opherk C, Danek A, Ballard C, Herzog J, Dichgans M (2005) The pattern of cognitive performance in CADASIL: a monogenic condition leading to subcortical ischemic vascular dementia. Am J Psychiatry 162, 2078-2085. 
[18] Buffon F, Porcher R, Hernandez K, Kurtz A, Pointeau S, Vahedi K, Bousser M-G, Chabriat H (2006) Cognitive profile in CADASIL. J. Neurol. Neurosurg. Psychiatry 77, 175-180.

[19] Charlton RA, Morris RG, Nitkunan A, Markus HS (2006) The cognitive profiles of CADASIL and sporadic small vessel disease. Neurology 66, 1523-1526.

[20] Proust C, Jacqmin-Gadda H, Taylor JMG, Ganiayre J, Commenges D (2006) A nonlinear model with latent process for cognitive evolution using multivariate longitudinal data. Biometrics 62, 1014-1024.

[21] Barbizet J, Duizabo P (1977) Abrégé de neuropsychologie, Masson, Paris ; New York.

[22] Stram DO, Lee JW (1994) Variance Components Testing in the Longitudinal Mixed Effects Model. Biometrics 50, 1171.

[23] R Core Team (2018) R: A Language and Environment for Statistical Computing, R Foundation for Statistical Computing, Vienna, Austria.

[24] Proust-Lima C, Philipps V, Liquet B (2017) Estimation of Extended Mixed Models Using Latent Classes and Latent Processes: The $R$ Package Icmm. Journal of Statistical Software 78,.

[25] Jacqmin-Gadda H, Fabrigoule C, Commenges D, Dartigues JF (1997) A 5-year longitudinal study of the Mini-Mental State Examination in normal aging. Am. J. Epidemiol. 145, 498-506.

[26] Mura T, Proust-Lima C, Jacqmin-Gadda H, Akbaraly TN, Touchon J, Dubois B, Berr C (2014) Measuring cognitive change in subjects with prodromal Alzheimer's disease. J. Neurol. Neurosurg. Psychiatry 85, 363-370.

[27] Legdeur N, Heymans MW, Comijs HC, Huisman M, Maier AB, Visser PJ (2018) Age dependency of risk factors for cognitive decline. BMC Geriatr 18, 187. 
[28] Proust-Lima C, Amieva H, Dartigues J-F, Jacqmin-Gadda H (2007) Sensitivity of four psychometric tests to measure cognitive changes in brain aging-population-based studies. Am. J. Epidemiol. 165, 344-350.

[29] Mura T, Proust-Lima C, Jacqmin-Gadda H, Akbaraly TN, Touchon J, Dubois B, Berr C (2014) Measuring cognitive change in subjects with prodromal Alzheimer's disease. J. Neurol. Neurosurg. Psychiatry 85, 363-370.

[30] Feng L, Li J, Yap K-B, Kua E-H, Ng T-P (2009) Vitamin B-12, apolipoprotein E genotype, and cognitive performance in community-living older adults: evidence of a gene-micronutrient interaction. Am. J. Clin. Nutr. 89, 1263-1268.

[31] Rolland Y, Abellan van Kan G, Nourhashemi F, Andrieu S, Cantet C, GuyonnetGillette S, Vellas B (2009) An abnormal "one-leg balance" test predicts cognitive decline during Alzheimer's disease. J. Alzheimers Dis. 16, 525-531. 


\section{LEGENDS}

Tables

Table 1. Baseline characteristics of the patients and comparison of patients included and not included in the analysis.

Table 2. Estimated fixed effects of the multivariate latent process mixed model in the latent process scale.

Table 3. Estimated interactions between the slopes and the characteristics at baseline.

\section{Figures}

Figure 1. Predicted trajectories in the scale of the latent process underlying the MMSE and the MDRS according to the educational level.

Figure 2. Estimated link functions between each test and the common underlying latent process with $95 \%$ confidence bands. 
Table 1. Baseline characteristics of the patients and comparison of patients included and not included in the analysis.

\begin{tabular}{lccc}
\hline Baseline characteristic & Included $(\mathrm{n}=185)$ & Not included $(\mathrm{n}=181)$ & P-value \\
\hline Age (years) & $50.32(11.54)$ & $54.33(12.12)$ & \\
Mean $(\mathrm{SD} *)$ & $185(0)$ & $180(1)$ & 0.001 \\
N $(\mathrm{NA} \dagger)$ & & & \\
Sex & & & \\
Male & $80(43.2 \%)$ & $89(49.4 \%)$ & 0.235 \\
NA & 0 & 1 &
\end{tabular}

Education

> high school diploma

$77(41.6 \%)$

$37(46.8 \%)$

0

0.434

NA

102

Smoking

Never

$70(43.5 \%)$

$60(42.3 \%)$

Former

$54(33.5 \%)$

$51(35.9 \%)$

Current

$37(23.0 \%)$

$31(21.8 \%)$

0.908

NA

24

39

Alcohol consumption

Never

$62(38.8 \%)$

$48(34.3 \%)$

$<2$ glasses of wine for a man

$80(50.0 \%)$

$79(56.4 \%)$

$>2$ glasses of wine for a man

$18(11.3 \%)$

$13(9.3 \%)$

NA

25

41

Hypertension

Yes

$33(20.4 \%)$

$45(31.7 \%)$

0.024 
Diabetes

Yes

$$
6(3.7 \%)
$$

NA

Systolic blood pressure $(\mathrm{mmHg})$

Mean (SD)

$\mathrm{N}$ (NA)

Diastolic blood pressure $(\mathrm{mmHg})$

Mean (SD)

$\mathrm{N}(\mathrm{NA})$

Homocysteine ( $\mu \mathrm{mol} / \mathrm{L})$

Mean (SD)

$\mathrm{N}(\mathrm{NA})$

Previous stroke events

Yes

Gait disturbance

Yes

NA

Balance problems

Yes

NA

Disability

Moderate or severe
22

$74.15(10.48)$

179 (6)

$96(51.9 \%)$

$99(55.6 \%)$

0

3
$11.38(3.74)$

$161(24)$

$131(50)$
0.050

38

130.65 (18.69)

175 (6)

0.063

75.83 (11.86)

175 (6)

0.158

0.011

0.477

$25(13.5 \%)$

0

$78(43.8 \%)$

3

$<0.001$

$38(20.5 \%)$

$67(37.6 \%)$

$<0.001$

0

3

$51(29.1 \%) \quad<0.001$ 


\begin{tabular}{lccc}
\hline Baseline characteristic & Included $(\mathrm{n}=185)$ & Not included $(\mathrm{n}=181)$ & P-value \\
\hline NA & 0 & 6 & \\
Dementia & $7(3.8 \%)$ & $30(16.9 \%)$ & \\
Yes & 0 & 4 & $<0.001$ \\
NA & $27.8(2.4)$ & $25.04(5.96)$ & $<0.001$ \\
MMSE & $184(1)$ & $159(22)$ & \\
Mean (SD) & & $127.51(23.25)$ & \\
N (NA) & $137.81(8.86)$ & $154(27)$ & \\
MDRS & $183(2)$ & & \\
Mean (SD) & & & \\
N (NA) & & & \\
\hline
\end{tabular}

* SD: standard deviation

$\dagger$ NA: number of missing data

The groups were compared using Pearson's Chi-squared test when the variables were qualitative and Student's t-test when the variables were quantitative. 
Table 2. Estimated fixed effects of the multivariate latent process mixed model in the latent process scale.

\begin{tabular}{|c|c|c|c|}
\hline Parameter & Estimate & $\mathrm{SE}^{*}$ & P-value \\
\hline Intercept $\dagger$ & 0.00 & & \\
\hline Linear slope & 0.76 & 0.33 & 0.019 \\
\hline Quadratic slope & -0.23 & 0.09 & 0.012 \\
\hline Sex: male & -0.30 & 0.20 & 0.136 \\
\hline Education: > high school diploma & 1.01 & 0.43 & 0.019 \\
\hline \multicolumn{4}{|l|}{ Contrasts for sex } \\
\hline On the MMSE & 0.14 & 0.08 & 0.085 \\
\hline On the MDRS $\ddagger$ & -0.14 & 0.08 & 0.085 \\
\hline \multicolumn{4}{|l|}{ Contrasts for education } \\
\hline On the MMSE & 0.09 & 0.09 & 0.283 \\
\hline On the MDRS $\ddagger$ & -0.09 & 0.09 & 0.283 \\
\hline
\end{tabular}

$\dagger$ The intercept was not estimated. Indeed, two constraints are required to obtain an identified multivariate latent process mixed model: the intercept is equal to 0 and the variance of the random intercept is equal to 1 .

$\ddagger$ The contrasts for sex and education on the MDRS were not estimated but obtained from the estimations of the contrasts for sex and education on the MMSE because the sum of the contrasts for a given covariate is equal to 0 .

In the first part of the table (above the dashed line), the estimates relate to the first part of the multivariate latent process mixed model that is the structural model. In the second part of the table (below the dashed line), the estimates relate to the 
second part of the multivariate latent process mixed model that is the measurement models. The estimates of the parameters of the two nonlinear transformations that relate the scores on each neuropsychological test with the common latent process are not provided. 
Table 3. Estimated interactions between the slopes and the characteristics at baseline.

\begin{tabular}{|c|c|c|c|}
\hline Baseline characteristic & $\begin{array}{l}\text { Interaction with the } \\
\text { linear slope }(\mathrm{SE} *)\end{array}$ & $\begin{array}{l}\text { Interaction with the } \\
\text { quadratic slope (SE) }\end{array}$ & $\mathrm{P}$-value $\dagger$ \\
\hline $\begin{array}{l}\text { Age at inclusion } \\
\text { (per } 10 \text { years increase) }\end{array}$ & $0.60(0.23)$ & $-0.02(0.03)$ & $<0.001$ \\
\hline Sex: male & $0.65(0.52)$ & $-0.15(0.10)$ & 0.104 \\
\hline Education: > high school diploma & $0.52(0.48)$ & $-0.07(0.09)$ & 0.344 \\
\hline Smoking & & & \\
\hline Never & 0.00 & 0.00 & \\
\hline Former & $-0.66(0.67)$ & $0.14(0.13)$ & 0.283 \\
\hline Current & $-1.01(0.74)$ & $0.15(0.14)$ & \\
\hline Alcohol consumption & & & \\
\hline Never & 0.00 & 0.00 & \\
\hline$<2$ glasses of wine for a man & $0.51(0.43)$ & $-0.12(0.08)$ & 0.095 \\
\hline$>2$ glasses of wine for a man & $-0.52(0.82)$ & $0.15(0.16)$ & \\
\hline Hypertension & $-0.04(0.82)$ & $-0.01(0.14)$ & 0.905 \\
\hline Diabetes & $1.62(1.99)$ & $-0.31(0.33)$ & 0.463 \\
\hline $\begin{array}{l}\text { Systolic blood pressure } \\
\text { (per } 10 \mathrm{mmHg} \text { increase) }\end{array}$ & $0.25(0.16)$ & $-0.03(0.03)$ & 0.131 \\
\hline $\begin{array}{l}\text { Diastolic blood pressure } \\
\text { (per } 10 \mathrm{mmHg} \text { increase) }\end{array}$ & $0.09(0.12)$ & $-0.02(0.03)$ & 0.883 \\
\hline Homocysteine $(\mu \mathrm{mol} / \mathrm{L})$ & $0.17(0.09)$ & $-0.03(0.02)$ & 0.058 \\
\hline Previous stroke events & $0.50(0.56)$ & $-0.09(0.10)$ & 0.586 \\
\hline Gait disturbances & $0.90(1.25)$ & $-0.19(0.19)$ & 0.258 \\
\hline
\end{tabular}




\begin{tabular}{lccc}
\hline Baseline characteristic & $\begin{array}{c}\text { Interaction with the } \\
\text { linear slope }(\mathrm{SE} *)\end{array}$ & $\begin{array}{c}\text { Interaction with the } \\
\text { quadratic slope (SE) }\end{array}$ & P-value $\dagger$ \\
\hline Balance problems & $0.16(0.70)$ & $-0.05(0.12)$ & 0.736 \\
Disability: moderate or severe & $5.62(3.82)$ & $-0.79(0.54)$ & 0.193 \\
Dementia & $6.54(3.71)$ & $-0.94(0.54)$ & 0.055 \\
\hline
\end{tabular}

* SE: standard error

$\dagger$ For each covariate, the p-value is derived from the likelihood ratio test involving the model that includes this covariate at baseline and the model that includes this covariate at baseline as well as the interaction between this covariate and the slope and the interaction between this covariate and the quadratic slope. 
Figure 1. Predicted trajectories in the scale of the latent process underlying the MMSE and the MDRS according to the educational level.

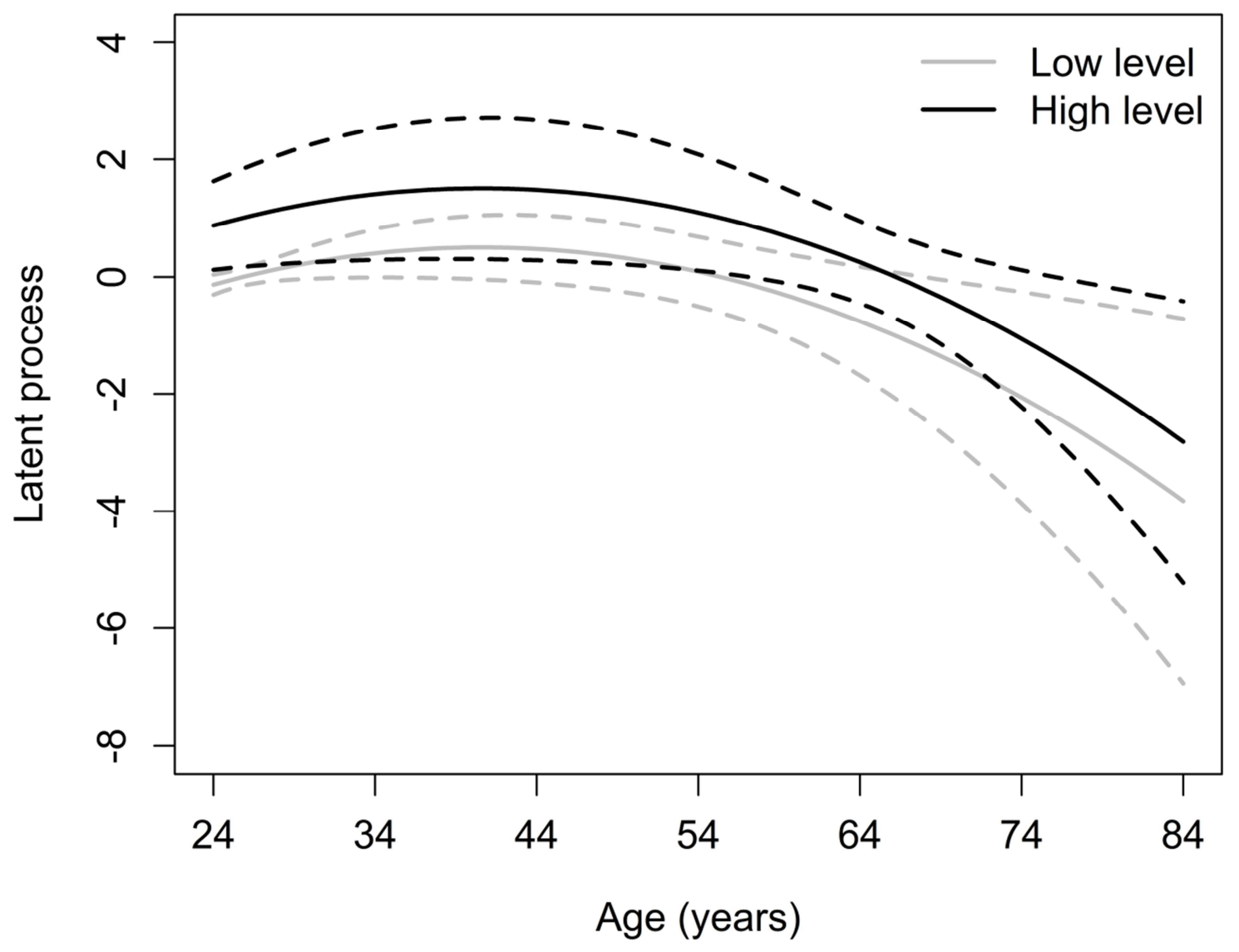

The structural model was $y=0.76 t-0.23 t^{2}+1.01$ for subjects with at least the high school diploma and $y=0.76 t-0.23 t^{2}$ for subjects who did not have the high school diploma. 
Figure 2. Estimated link functions between each test and the common underlying latent process with $95 \%$ confidence bands.

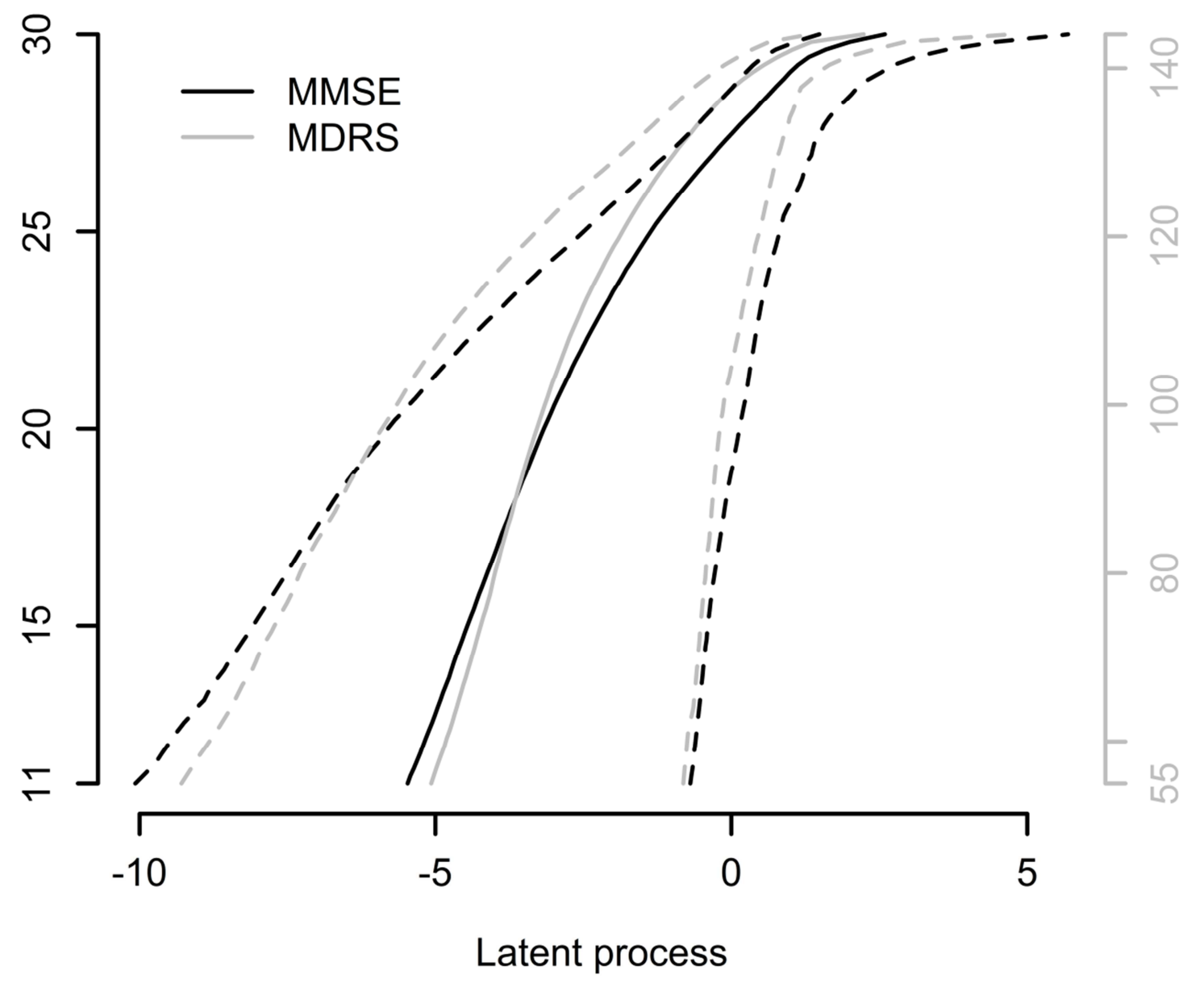


SUPPLEMENTARY MATERIAL

Supplementary Tables

Supplementary Table 1. Numbers of observations and patients per age range.

\begin{tabular}{lcc}
\hline Age range & Number of observations & \multicolumn{2}{c}{ Number of patients } \\
\hline$[24,29)$ & 14 & 6 \\
{$[29,34)$} & 36 & 17 \\
{$[34,39)$} & 47 & 26 \\
{$[39,44)$} & 71 & 39 \\
{$[44,49)$} & 116 & 63 \\
{$[49,54)$} & 132 & 68 \\
{$[54,59)$} & 126 & 73 \\
{$[59,64)$} & 126 & 62 \\
{$[64,69)$} & 95 & 49 \\
{$[69,74)$} & 59 & 32 \\
{$[74,79)$} & 18 & 12 \\
{$[79,84)$} & 1 & 1 \\
\hline
\end{tabular}


Supplementary Table 2. Selection of the best univariate models.

Link function Number of parameters

$\mathrm{AIC} *$

\begin{tabular}{lccc}
\hline & & MMSE & MDRS \\
\hline Linear & 15 & 3697.87 & 5547.76 \\
Beta CDF $\dagger$ & 17 & 4355.45 \\
Quadratic I-splines with three nodes located at quantiles $\ddagger$ & 18 & 2934.85 & 4448.69 \\
Quadratic I-splines with five nodes located at quantiles § & 20 & - & 4335.22 \\
Quadratic I-splines with three equidistant nodes || & 18 & 3264.70 & 4879.51 \\
Quadratic I-splines with five equidistant nodes \# & 20 & 3167.55 & 4727.36 \\
Quadratic I-splines with seven equidistant nodes ** & 22 & 3103.06 & 4661.58
\end{tabular}

* AIC: Akaike information criterion

$\dagger$ The maximum number of iteration was reached without convergence for the MMSE.

$\$$ Three nodes located at the $0^{\text {th }}, 50^{\text {th }}$, and $100^{\text {th }}$ quantiles corresponding to MMSE scores $11.00,29.00$, and 30.00 and MDRS scores 55.00, 141.00, and 144.00.

$\S$ Five nodes located at the $0^{\text {th }}, 25^{\text {th }}, 50^{\text {th }}, 75^{\text {th }}$, and $100^{\text {th }}$ quantiles corresponding to MMSE scores 11.00 , 27.00, 29.00, 30.00, and 30.00 and MDRS scores 55.00, 135.00, 141.00, 143.00, and 144.00. The link function could not be estimated for MMSE since some nodes were equal.

|| Three equidistant nodes corresponding to MMSE scores 11.00, 20.50, and 30.00 and MDRS scores 55.00, 99.50, and 144.00 .

\# Five equidistant nodes corresponding to MMSE scores 11.00, 15.75, 20.50, 25.25, and 30.00 and MDRS scores $55.00,77.25,99.50,121.75$, and 144.00 .

** Seven equidistant nodes corresponding to MMSE scores 11.00, 14.17, 17.33, 20.50, 23.67, 26.83, and 30.00 and MDRS scores 55.00, 69.83, 84.67, 99.50, 114.33, 129.17, and 144.00. 
Supplementary Table 3. Selection of the best multivariate model.

\begin{tabular}{|c|c|c|c|c|c|c|c|c|}
\hline Model & Fixed effects & Random effects & $\begin{array}{c}\text { Matrix of variance- } \\
\text { covariance }\end{array}$ & $\begin{array}{c}\text { Autocorrelated } \\
\text { process }\end{array}$ & $\begin{array}{l}\text { Test-specific } \\
\text { random effects }\end{array}$ & Covariates & $-2 \mathrm{LL} *$ & $\mathrm{AIC} \dagger$ \\
\hline 1 & $\begin{array}{l}\text { Linear, quadratic, and } \\
\text { cubic slopes }\end{array}$ & $\begin{array}{l}\text { Linear, quadratic, } \\
\text { and cubic slopes }\end{array}$ & Unstructured & No & No & None & 7044.22 & 7096.23 \\
\hline $2 \div$ & $\begin{array}{l}\text { Linear, quadratic, and } \\
\text { cubic slopes }\end{array}$ & $\begin{array}{c}\text { Linear and } \\
\text { quadratic slopes }\end{array}$ & Unstructured & No & No & None & 7057.56 & 7101.56 \\
\hline 3 & $\begin{array}{l}\text { Linear, quadratic, and } \\
\text { cubic slopes }\end{array}$ & Linear slope & Unstructured & No & No & None & 7097.98 & 7135.98 \\
\hline 4 & $\begin{array}{l}\text { Linear, quadratic, and } \\
\text { cubic slopes }\end{array}$ & $\begin{array}{c}\text { Linear and } \\
\text { quadratic slopes }\end{array}$ & Diagonal & No & No & None & 7097.19 & 7135.19 \\
\hline 5 & $\begin{array}{l}\text { Linear, quadratic, and } \\
\text { cubic slopes }\end{array}$ & $\begin{array}{c}\text { Linear and } \\
\text { quadratic slopes }\end{array}$ & Unstructured & Brownian motion & No & None & 7049.65 & 7095.65 \\
\hline $6 \S$ & $\begin{array}{l}\text { Linear, quadratic, and } \\
\text { cubic slopes }\end{array}$ & $\begin{array}{c}\text { Linear and } \\
\text { quadratic slopes }\end{array}$ & Unstructured & $\begin{array}{c}\text { Autoregressive } \\
\text { process }\end{array}$ & No & None & - & - \\
\hline
\end{tabular}




\begin{tabular}{|c|c|c|c|c|c|c|c|c|}
\hline Model & Fixed effects & Random effects & $\begin{array}{c}\text { Matrix of variance- } \\
\text { covariance }\end{array}$ & $\begin{array}{c}\text { Autocorrelated } \\
\text { process }\end{array}$ & $\begin{array}{l}\text { Test-specific } \\
\text { random effects }\end{array}$ & Covariates & $-2 \mathrm{LL} *$ & $\mathrm{AIC} \dagger$ \\
\hline $7 \ddagger$ & $\begin{array}{c}\text { Linear and } \\
\text { quadratic slopes }\end{array}$ & $\begin{array}{c}\text { Linear and } \\
\text { quadratic slopes }\end{array}$ & Unstructured & Brownian motion & No & None & 7053.38 & 7097.38 \\
\hline 8 & $\begin{array}{c}\text { Linear and } \\
\text { quadratic slopes }\end{array}$ & $\begin{array}{c}\text { Linear and } \\
\text { quadratic slopes }\end{array}$ & Unstructured & Brownian motion & Yes & None & 7007.64 & 7055.64 \\
\hline 9 & $\begin{array}{c}\text { Linear and } \\
\text { quadratic slopes }\end{array}$ & $\begin{array}{c}\text { Linear and } \\
\text { quadratic slopes }\end{array}$ & Unstructured & Brownian motion & Yes & $\begin{array}{l}\text { Sex and } \\
\text { education }\end{array}$ & 6960.24 & 7016.24 \\
\hline
\end{tabular}

* LL: maximum log-likelihood.

$\dagger$ AIC: Akaike information criterion

\$ The p-value of the likelihood ratio test (LRT) involving models 1 and 2 was 0.007 but the fixed cubic effect of time was not significant, which led to the selection of model 2. The p-value of the LRT involving models 2 and 3 was $<0.001$, which led to the selection of model 2 . The p-value of the LRT involving models 5 and 7 was 0.054 , which led to the selection of model 7 .

$\S$ The model with an autoregressive process did not converge.

To link the common latent process with each score, we used quadratic I-splines with 3 nodes at the quantiles of the MMSE distribution and quadratic Isplines with 5 nodes at the quantiles of the MDRS distribution. 


\section{Supplementary Figures}

Supplementary Figure 1. Distribution of the repeated measures of MMSE and MDRS sum scores among the 185 subjects included in the analysis.
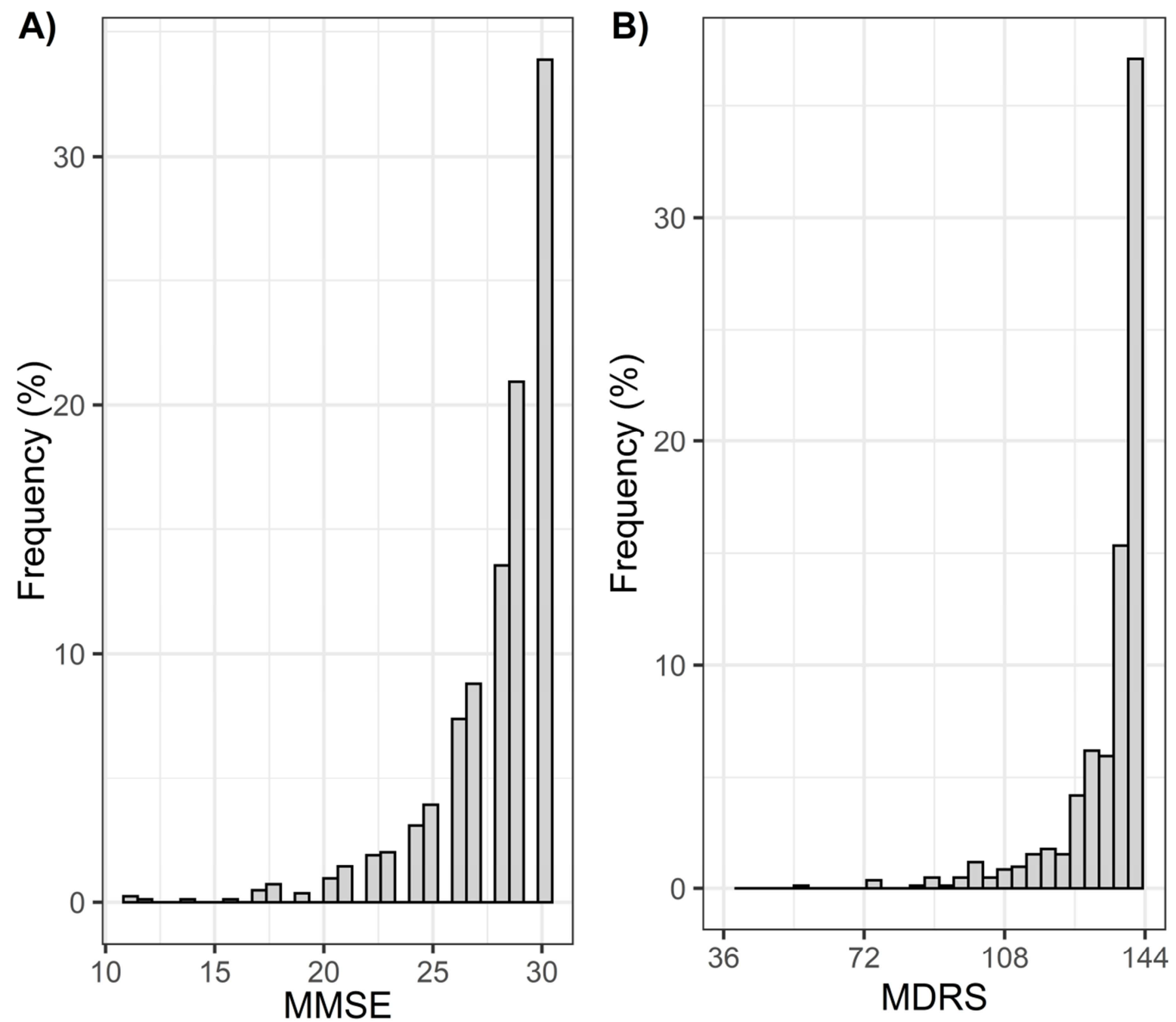
Supplementary Figure 2. Weighted marginal predictions versus observed evolution for MMSE and MDRS tests.

A)

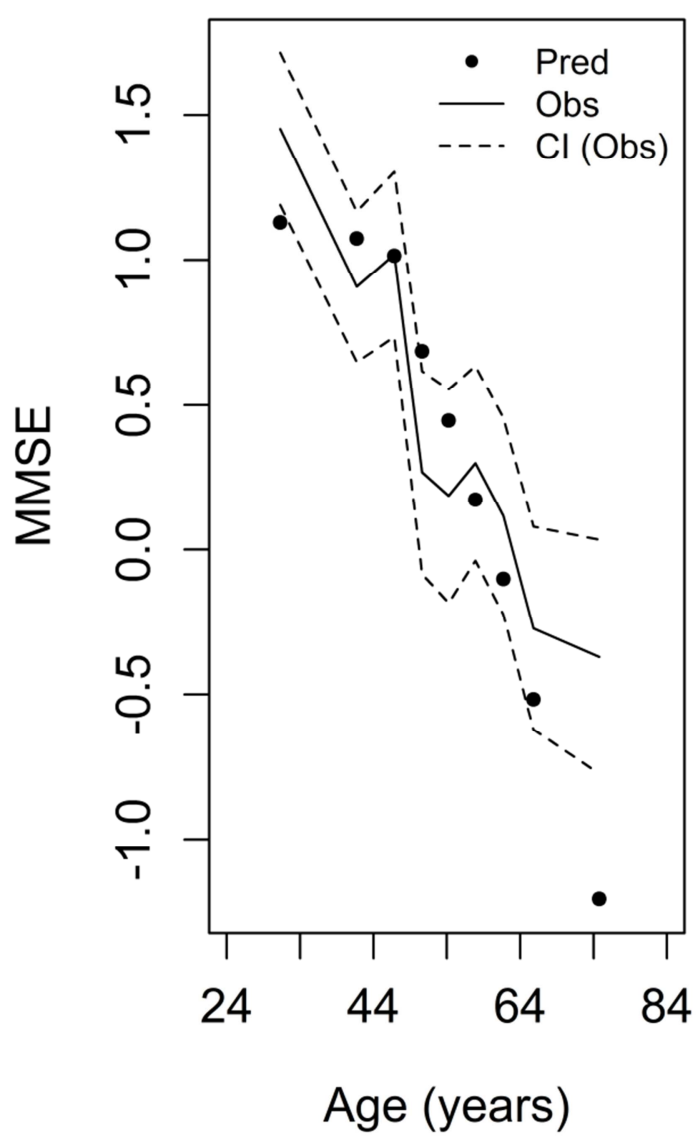

B)

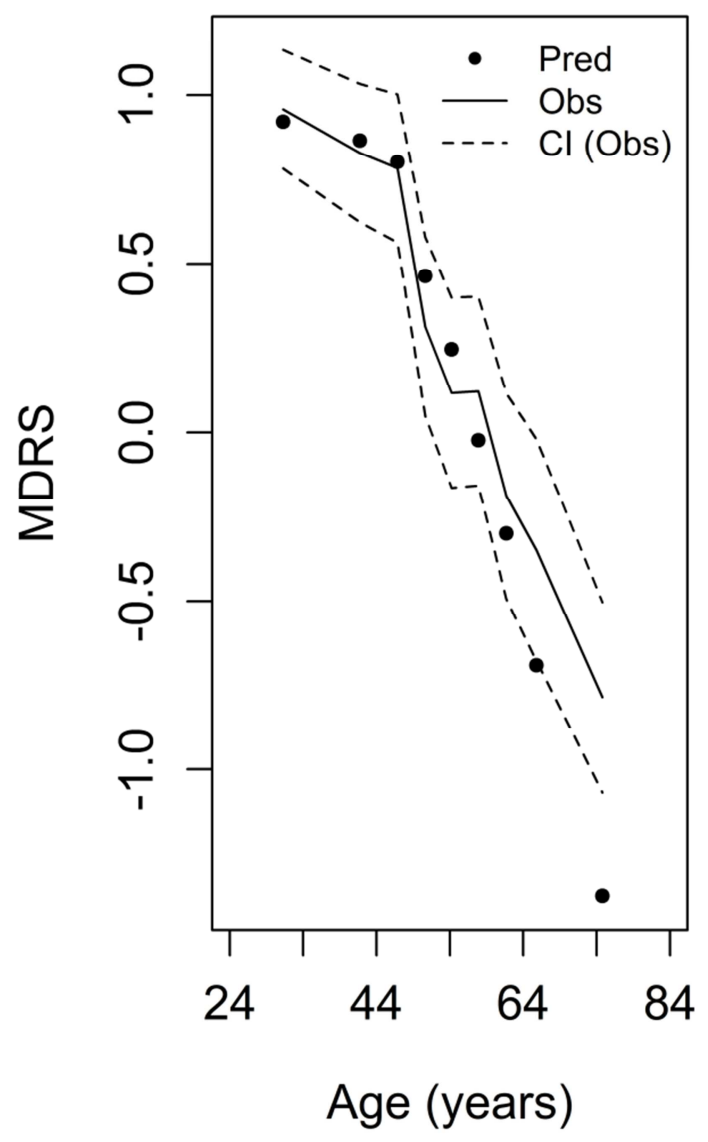

Pred: predictions obtained with the multivariate latent process mixed model described in

Table 2, Obs: observed evolution, CI: confidence interval of observed evolution 
Supplementary Figure 3. Percentage of variance explained by the latent process for each test according to age.

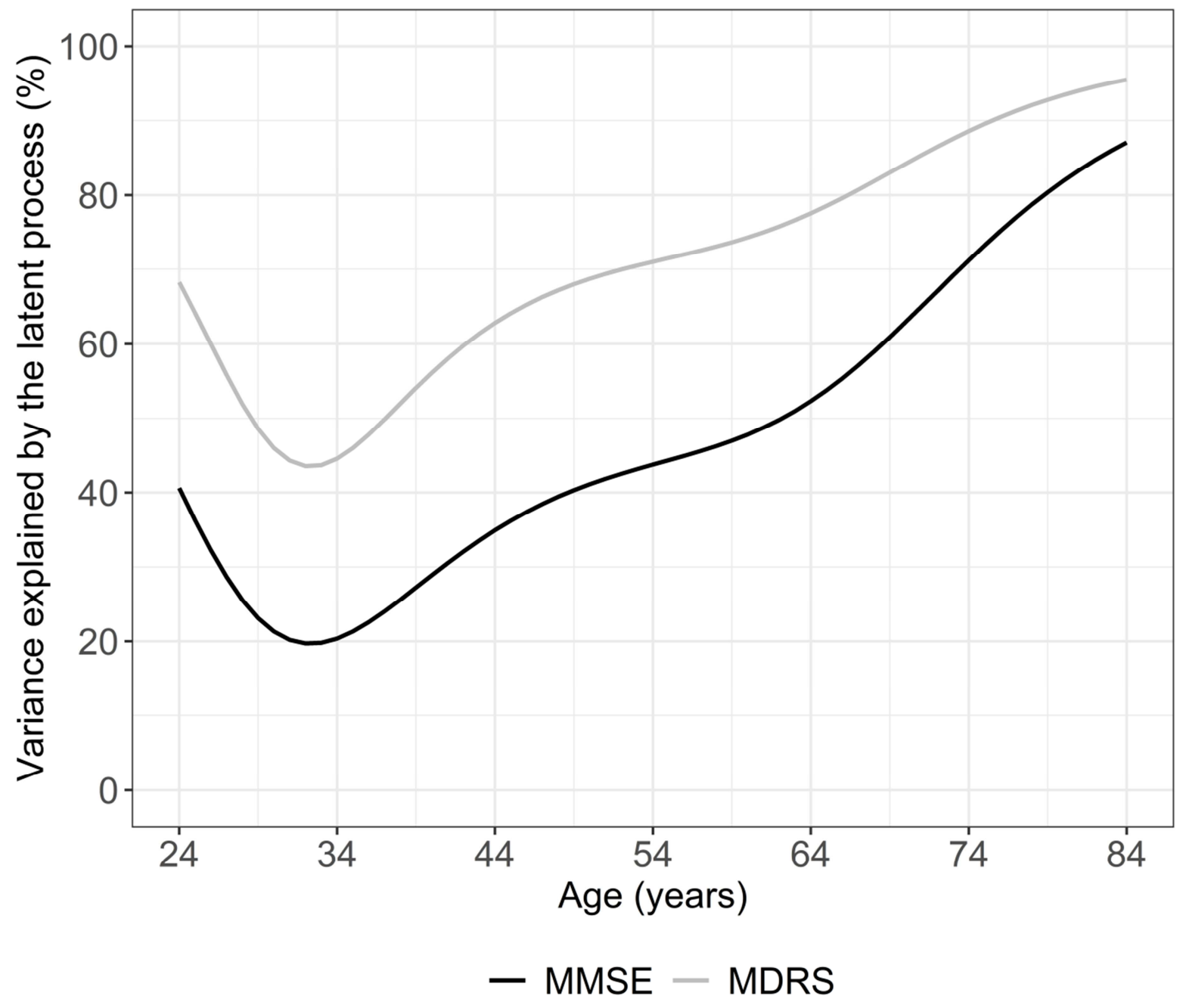

